\title{
Validity of Intraepithelial Lymphocyte Count in the Diagnosis of Celiac Disease: A Histopathological Study
}

\author{
Eranga Himalee Siriweera ${ }^{1, *}$, Zhengyan $\mathbf{Q i}^{2}$, Jim. L. C. Yong ${ }^{2}$ \\ ${ }^{1}$ Department of Pathology, Faculty of Medicine, University of Peradeniya, Peradeniya, Sri Lanka \\ ${ }^{2}$ Department of Anatomical Pathology, Liverpool hospital, New South Wales, Australia \\ *Corresponding author: eranga.siriweera@gmail.com
}

Received October 05, 2015; Revised October 12, 2015; Accepted October 13, 2015

\begin{abstract}
The gold standard for diagnosis of Celiac disease (CD) is histological evidence of a small intestinal biopsy together with positive serology. Modified Marsh classification utilizes the histological parameter of intraepithelial lymphocyte (IEL) count in the diagnosis of CD. The reported upper limit of normal IEL in the duodenum varies from 20-40 per 100 epithelial cells (EC). The objectives of the study are to determine the normal upper limit of IEL in the duodenum and assess the diagnostic accuracy of existing criteria of IEL counts to diagnose $\mathrm{CD}$. A retrospective analysis of histopathological records of duodenal biopsies reported as normal (control group, $\mathrm{n}=38)$ and consistent with $\mathrm{CD}(\mathrm{n}=37)$ formed the basis of the study. IEL were counted in an uninterrupted length of surface epithelium and villous tips from formalin fixed, paraffin embedded, Haematoxylin and Eosin stained biopsies under the light microscope (X400 magnification). In the control group the mean IEL/100EC was 3.81(upper limit of normal $=7.78$ ) and the mean IEL/villous tip was 0.96 (upper limit of normal $=3.5$ ). In CD the mean IEL/100EC and mean IEL/villous tip were 20.93 and 6.83 respectively. The upper limit of normal IEL/100EC, mean IEL/100EC in CD and the villous tip IEL count in both the control and CD groups were considerably lower than those reported in other studies. The ethnicity, country of origin and environmental factors may be partly responsible for this observation. If high cut off values of IEL/100EC is taken to diagnose CD many cases may be under diagnosed, particularly when the upper limit of the normal IEL count is lower for that population and region, highlighting the importance of a multidisciplinary approach in the diagnosis of CD.
\end{abstract}

Keywords: Celiac disease, duodenal biopsy, Marsh classification, Intraepithelial lymphocytes, Villous tip

Cite This Article: Eranga Himalee Siriweera, Zhengyan Qi, and Jim. L. C. Yong, "Validity of Intraepithelial Lymphocyte Count in the Diagnosis of Celiac Disease: A Histopathological Study." International Journal of Celiac Disease, vol. 3, no. 4 (2015): 156-158. doi: 10.12691/ijcd-3-4-7.

\section{Introduction}

Intraepithelial lymphocytes (IELs) are a normal constituent of the small intestinal mucosa where they play a significant role in immune surveillance and activation. Celiac disease (CD) is a chronic enteropathy affecting the small bowel. The combination of clinical, serologic, histological, genetic findings and a response to a gluten free diet confirms the diagnosis of CD [1,2].

Small intestinal biopsy together with positive serology is the gold standard for the diagnosis of CD and the IEL count is a key parameter which is assessed histologically to aid the diagnosis[1]. The upper limit of normal IEL per 100 epithelial cells (EC) in the proximal small intestine has been reported to vary from 20-40 [1,3,4,5,6,7,8]. A consensus on the normal IEL count is lacking. A more practical, fast and easy method of counting villous tip lymphocytes have been used to diagnose potential CD and it is sufficient for making a diagnosis of increased IELs [9].

The objectives of the study are to determine the normal upper limit of IEL in the small intestine and to assess the diagnostic accuracy of existing criteria for IEL counts to diagnose $\mathrm{CD}$.

\section{Materials and Method}

The data was collected from retrospective analysis of the histopathology records of duodenal biopsies received in the Department of Anatomical Pathology, Liverpool Hospital Australia between 2009 and 2011. The control group comprising 38 subjects was randomly selected from patients presenting with upper gastrointestinal symptoms from whom a duodenal biopsy was obtained as a routine procedure and was reported as normal. The study group of 37 subjects was randomly selected from those who had a clinical suspicion of CD with biopsy reports consistent with CD.

Demographic data and clinical history were collected from the records. All biopsies were fixed in $10 \%$ buffered formalin, embedded in paraffin wax, sections of $3 \mu \mathrm{m}$ thickness were cut at three levels and stained with Haematoxylin and Eosin (H \& E). Well oriented sections were examined by light microscopy (x400 magnification) and the number of IELs in a randomly chosen, 
uninterrupted length of surface (villous) epithelium comprising 500 cells was counted. Only perinuclear or supranuclear lymphocytes above the basement membrane of villi were assumed as IEL (Figure 1). Counts based on 500 EC gave a mean for the number of IELs/100 cells. In addition the numbers of IEL in 20 EC in 5 consecutive villous tips were counted to determine the mean IEL/20 villous tip EC. The upper limit of normal IEL/100 EC and IEL/villous tip was determined by calculating the $97^{\text {th }}$ percentile. The diagnostic accuracy of existing criteria of IEL counts to diagnose CD was assessed by comparing with the IEL counts of the normal controls with those of CD.

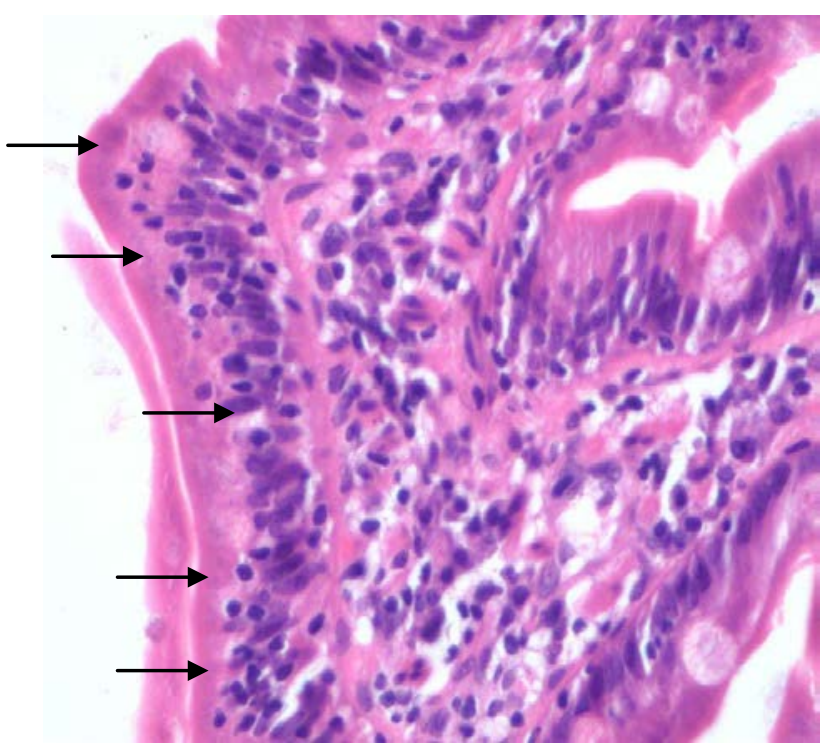

Figure 1. Duodenal biopsy in CD showing IEL, some of which are indicated by arrows ( $\mathrm{H} \& \mathrm{E} x \mathrm{400})$

\section{Results}

The control group consisted of 38 subjects with a mean age of 53.4 years (range, 19-86yrs). Of this 15 were males (mean age 50.7yrs) and 23 were females (mean age $55.2 \mathrm{yrs}$ ). The mean IEL/100 EC in this group was 3.81 (range 1-8.2). The upper limit of normal IEL/100 EC was $7.78\left(97^{\text {th }}\right.$ percentile). The mean IEL/villous tip was 0.96 (range 0-3.6) and the upper limit of normal IEL/villous tip was $3.5\left(97^{\text {th }}\right.$ percentile).

In the 37 subjects in whom a diagnosis consistent with CD was made the mean age was 44.39 years (range 1889yrs). This included 13 males (mean age-53.3yrs) and 24 females (mean age 39.9 yrs). A diagnosis of CD Marsh type 1,11 or 111 was given in 19 cases (Marsh1-6, Marsh11-5, Marsh 111-8) and the other 18 cases were diagnosed as consistent with CD. The mean IEL/100 EC was 20.69 and 21.12 in the group with CD Marsh type 1 to 111 and the group with a diagnosis consistent with CD respectively. Analysis using the Mann Whitney $U$ test revealed that there was no significant difference ( $p$-value 0.718) in the mean IEL/100 EC between these two groups. Therefore the two groups were analyzed as one to determine the mean IEL/100 EC in CD which was 20.93(range 8-38).

The mean IEL/villous tip was 6.83 (range 4-12.4), calculated in 29 cases in the group with CD excluding the Marsh type 111 lesions in which villous atrophy is identified. The results of IEL counts are summarized in Table 1.

Table 1. IEL counts in the control group and CD group

\begin{tabular}{|c|c|c|c|c|}
\hline & $\begin{array}{c}\text { Mean } \\
\text { IEL/100EC }\end{array}$ & $\begin{array}{c}\text { Upper limit } \\
\text { IEL/100EC }\end{array}$ & $\begin{array}{c}\text { Mean } \\
\text { IEL/vilous } \\
\text { tip }\end{array}$ & $\begin{array}{c}\text { Upper limit } \\
\text { IEL/villous } \\
\text { tip }\end{array}$ \\
\hline $\begin{array}{c}\text { Control } \\
\text { group }\end{array}$ & 3.81 & 7.78 & 0.96 & 3.5 \\
\hline $\begin{array}{c}\text { CD } \\
\text { group }\end{array}$ & 20.93 & NA & 6.83 & NA \\
\hline
\end{tabular}

NA: Not Applicable.

\section{Discussion}

CD occurs in genetically predisposed individuals on exposure to gluten containing diets and results in an immunologically mediated chronic enteropathy affecting the small intestine [1]. Increased numbers of IEL is the earliest and a sensitive pathologic change seen in the small bowel in CD.

The upper limit of normal IEL/100 EC in this study was 7.78 which was considerably lower than that reported in other studies. The upper normal limit of IELs in the proximal small intestine has long been considered to be 40 IELs per 100 EC, a ratio used as a criterion of the MarshOberhuber classification for gluten sensitivity [3]. This figure was derived by counts performed on thickly sectioned $(7-\mu \mathrm{m})$ Hematoxylin and Eosin stained sections [4]. However, recent studies employing more thinly cut sections of $3 \mu \mathrm{m}$ and $4 \mu \mathrm{m}$ and CD3 immunohistochemistry have observed the upper limit of normal in the proximal small intestine to be as low as 20 IEL/100 EC on hematoxylin-eosin stained sections and 25 IEL/100 EC with immunohistochemistry [5,6]. Mahadewa et al. reported 22 IEL as the upper limit of normal [8].

The mean IEL/100 EC in cases diagnosed with CD in the studied group was 20.93 (range 8-38). The modified Marsh criteria utilizes more than 30 IEL/100EC in the diagnosis of CD (Table 2) [1].

Table 2. Modified Marsh Classification of histologic findings in CD

\begin{tabular}{|c|c|c|c|}
\hline $\begin{array}{c}\text { Marsh } \\
\text { Type }\end{array}$ & $\begin{array}{c}\text { IEL / } 100 \\
\text { enterocytes - } \\
\text { duodenum }\end{array}$ & $\begin{array}{c}\text { Crypt } \\
\text { hyperplasia }\end{array}$ & Villi \\
\hline 0 & $<30$ & Normal & Normal \\
\hline 1 & $>30$ & Normal & Normal \\
\hline 2 & $>30$ & Increased & Normal \\
\hline 3a & $>30$ & Increased & Mild atrophy \\
\hline 3b & $>30$ & Increased & Marked atrophy \\
\hline 3c & $>30$ & Increased & Complete atrophy \\
\hline
\end{tabular}

When values more than 30 IEL/100EC is taken to diagnose CD many cases may be under diagnosed, particularly when the upper limit of the normal IEL count is lower for that population and region than that of the value suggested in the diagnostic criteria.

In this study the mean normal IEL/villous tip was 0.96(range 0-3.6) and the upper limit of normal is 3.5 IEL/villous tip. The mean IEL/villous tip in CD was 6.83(range 4-12.4). A similar study revealed a mean villous tip IEL count of 4.6 (range, 1.4-7.8) in non-coeliac controls, 7.9 (range, 2.0-18.6) in treated CD, and 9.2 (range 5.8-21.8) in patients with potential CD [9]. The 
numbers in our study are lower in both the control group and in patients with CD in keeping with the lower counts seen when IEL/100EC was analyzed. As quoted by Ensari, Jarvinen et al has reported a normal mean number of IELs/villous tip of less than 5, while counts between 6 to 12 per 20 EC are considered suggestive of CD [7]. A value of 6.83 IEL/villous tip observed in this study falls within the range specified but is at the lower end of the spectrum.

\section{Conclusion}

The upper limit of normal IEL/100 EC was 7.78 and the mean IEL/100 EC in CD is 20.93, which are much lower than the values reported in the literature. The ethnicity and origin of the subjects and environmental factors may be partly responsible for this observation. To minimize the effects of possible regional and ethnic variation in IEL counts, a multidisciplinary approach taking in to account the clinical details, serology, biopsy findings and genetic tests where available will improve the diagnosis of CD. Counting villous tip IEL appear to be more practical in routine histopathology reporting when combined with other diagnostic parameters.

\section{References}

[1] World gastroenterology organization, Global guidelines, Celiac Disease, 2012. [Online] Available: http://www.worldgastroenterology.org/guidelines/globalguidelines/celiac-disease.

[2] Caio G, Volta U. Coeliac Disease: Changing diagnostic criteria? Gastroenterol Hepatol Bed Bench, 5. 119-122. 2012.

[3] Antonioli D. Coeliac disease :A progress report. Mod Pathol, 6(4). 342-346. 2003.

[4] Ferguson A, Murray D. Quantitation of intraepithelial lymphocytes in human jejunum. Gut, 12. 988-994. 1971.

[5] Hayat M, Cairns A, Dixon MF, O’Mahony S. Quantitation of intraepithelial lymphocytes in human duodenum: what is normal? J Clin Pathol. 55. 393-395. 2002.

[6] Brown I, Mino-Kenudson M, Deshpande V, Lauwers GY, Intraepithelial Lymphocytosis in Architecturally Preserved Proximal Small Intestinal Mucosa. An Increasing Diagnostic Problem with a Wide Differential Diagnosis. Arch Pathol Lab Med, 130. 1020-1025. 2006.

[7] Ensari A. Gluten-sensitive Enteropathy (Celiac Disease) Controversies in Diagnosis and Classification. Arch Pathol Lab Med, 134. 826-836. 2010.

[8] Mahadeva S, Wyatt JI, Howdle PD. Is a raised intraepithelial lymphocyte count with normal duodenal villus architecture clinically relevant? J Clin Pathol., 55. 424-428. 2002.

[9] Biagi F, Luinetti O, Campanello J, Klersy C et al. Intraepithelial Disease,J Clin Pathol ,57. 835-839. 2004. 